\title{
Has carotid intima-media thickness prognostic impact in patients with high cardiovascular risk? A long-term cohort study
}

\author{
Ana Teresa Timóteo $\mathrm{PhD}^{1,2}$ (D) | Miguel Mota Carmo PhD ${ }^{1,2}$ | Cristina Soares $\mathrm{RN}^{1}$ | \\ Rui Cruz Ferreira MD ${ }^{1,2}$
}

${ }^{1}$ Cardiology Department, Santa Marta Hospital, Centro Hospitalar Universitário de Lisboa Central, Lisbon, Portugal

${ }^{2}$ Centro de Estudos de Doenças Crónicas (CEDOC), NOVA Medical School, Lisbon, Portugal

Correspondence

Ana Teresa Timóteo, Cardiology

Department, Hospital Santa Marta, Lisboa, Portugal.

Email:ana_timoteo@yahoo.com
Background: Carotid intima-media thickness (CIMT) is an established surrogate marker for cardiovascular events in patients with intermediate risk. In patients with high cardiovascular risk or established cardiovascular disease, the impact of CMIT measurement on risk stratification for future events is less clear. Our objective was to evaluate the impact of CIMT on the occurrence of cardiovascular events in a cohort of individuals with high cardiovascular risk, in long-term follow-up.

Methods: We analyzed 296 individuals, mean follow-up of $6.9 \pm 2.2$ years. Individuals were divided into tertiles according to CIMT. Tertiles were compared in terms of baseline characteristics and outcomes during follow-up-all-cause mortality and composite outcome (mortality, acute coronary syndromes, coronary revascularization, stroke/transient ischemic attack, heart failure, or cardiovascular admission).

Results: Our population had a mean age of $65 \pm 9$ years at the beginning of the study, $55 \%$ males. Patients with higher CIMT showed a trend for higher cardiovascular mortality $(P=0.084)$ and for the composite outcome $(P=0.049)$. A CIMT $\geq 0.85 \mathrm{~mm}$ was also associated with higher rate of events; however, CIMT was not an independent predictor of outcome after adjustment for age and gender. CIMT assessment was useful in patients with hypertension, hyperlipidemia, and metabolic syndrome and in nondiabetic patients. For the composite outcome, it was also useful in females, smokers, and in patients without coronary artery disease.

Conclusions: Patients with higher CIMT have worst outcome, but this was mainly driven by age and gender. CIMT is useful as a prognostic marker in specific subsets of patients.

\section{KEYWORDS}

carotid intima-media thickness, coronary artery disease, prognosis

\section{1 | INTRODUCTION}

Cardiovascular diseases remain the leading cause of death in developed countries. ${ }^{1,2}$ In Portugal, cardiovascular diseases represent a major health problem, not only ischemic heart disease, but also stroke whose incidence in Portugal is among the highest in the world. According to National Statistics, although a 33\% decrease has been observed since 1990, deaths due to cardiovascular diseases still represented $29.7 \%$ of all deaths in 2015 in Portugal. ${ }^{3}$ In other developed countries, the most common cardiovascular disease is ischemic heart disease, which accounts for more than $20 \%$ of deaths in Europe. ${ }^{4}$ Clinically, the identification of patients 
at risk of developing stroke and other cardiovascular events is of great significance, enabling preventive interventions and lifestyle modifications. Carotid intima-media thickness (CIMT) has been suggested as a surrogate marker for coronary and peripheral artery disease. Being easily evaluated by a noninvasive test, it is recommended as "appropriate" in guidelines for cardiovascular risk stratification in the absence of known coronary artery disease for initial detection, particularly in patients at intermediate risk. $^{5}$ In patients with high cardiovascular risk or with established cardiovascular disease, the role of CIMT is less well defined and guidelines consider its use as "inappropriate or unknown." Few studies evaluated the prognostic value of CIMT in patients with established coronary artery disease and with conflicting results. ${ }^{6,7}$

In a previous study performed by our group, we sought to analyze the relationship between metabolic syndrome, coronary artery disease, and subclinical carotid atherosclerosis (by CIMT). ${ }^{8,9}$ At that time, we performed a cross-sectional analysis and included individuals were then prospectively followed for more than 5 years. Herein, we aimed to analyze the prognostic value of CIMT to predict fatal and nonfatal vascular events in long-term follow-up in the same high cardiovascular risk population of patients.

\section{2 | POPULATION AND METHODS}

The main study was an observational and cross-sectional study, with prospective inclusion of adult patients admitted for an elective coronary angiography due to suspected coronary artery disease (with stable angina and/or with documented ischemia by noninvasive tests). 8,9 Patients with previous acute coronary syndromes, myocardial revascularization procedure, valvular heart disease, congenital heart disease, cardiomyopathy, or stroke/transient ischemic attack (TIA) were excluded from the study. The local institutional ethics committee approved the study and all patients gave their written informed consent.

Blood pressure was measured on several occasions during hospital stay, and hypertension was defined by a previous diagnosis of hypertension or the presence of systolic blood pressure $\geq 140 \mathrm{~mm} \mathrm{Hg}$ or diastolic blood pressure $\geq 90 \mathrm{~mm} \mathrm{Hg}$ (mean of two consecutive measurements). Patients that smoked during the previous 6 months were classified as smokers and were selfreported. Diabetes was recorded by the investigator based on patient history, increased glucose (fasting level $\geq 126 \mathrm{mg} / \mathrm{dL}$ ), a glycated hemoglobin $>6.5 \%$, or concomitant use of specific therapy.

A venous blood sample was drawn after a 12-hour overnight fast. All the samples were analyzed in the hospital central laboratory. Blood glucose, total cholesterol, and triglycerides were determined using automatic standard routine enzymatic methods. HDL-cholesterol was determined after specific precipitation. LDLcholesterol was determined by Friedewald formula.

Coronary angiography was performed by the standard Judkins technique. The coronary angiograms were analyzed using
QCA software and Cardiovascular Measurement System (QCA$\mathrm{CMS}^{\mathrm{TM}}$ ) version 6.0 (Medis Medical Imaging Systems, Leiden, The Netherlands) by a single operator, blinded to the carotid ultrasound results. Significant angiographic coronary artery disease was defined by a stenosis of $70 \%$ or more in any coronary vessel (or $50 \%$ for left main lesions).

Carotid intima-media thickness was evaluated by a trained radiologist (blinded for the coronary angiography result) with a Siemens Sonolite $^{\mathrm{TM}}$ system and a $7.5-\mathrm{MHz}$ linear array transducer. Highresolution B-mode ultrasonography was used. In magnified and frozen images, CIMT was measured at the distal common carotid artery ( $1 \mathrm{~cm}$ proximal to the carotid bifurcation) at the far wall. Manual measurements of CIMT were obtained from both the right side and the left side. The lumen/intima leading edge to media/adventitia leading edge method was used. ${ }^{10} \mathrm{CIMT}$ value used for the present analysis was defined as the maximum value between the right and left common carotids. The interclass correlation coefficients for intra-reader and inter-reader reproducibility were 0.907 and 0.820 , respectively, which suggests good agreement beyond chance. ${ }^{8,9}$

Follow-up was obtained by telephone interview with the patient or with a close family member or by review of the medical records at 30 days, 1 year, and at least 5 years after inclusion in the study. A dedicated nursing team completed the follow-up. In the case of events during the follow-up period, patient's medical records were thoroughly analyzed (if the events occurred in our hospital). For events in other hospitals, a contact was made with the attending physician of that institution to correctly adjudicate events.

Our primary outcome was all-cause mortality. Other secondary endpoints were also analyzed: cardiovascular mortality, acute coronary syndrome, coronary revascularization after the index admission, heart failure, stroke/TIA, or cardiovascular hospital admission. A composite outcome of all the mentioned outcomes was specifically analyzed.

\section{1 | Statistical analysis}

Statistical analysis was performed using the PASW 18.0 program (SPSS Inc, Chicago, IL, USA). A P-value $<0.05$ was considered statistically significant.

Quantitative variables were described as mean and standard deviation, or as median and interquartile range for non-normally distributed variables. Normality was assessed with KolmogorovSmirnov test. Qualitative variables are presented as percentages. ANOVA test or Kruskal-Wallis test was used for between-group comparisons of continuous variables (according to distribution characteristics), while chi-square test was used for between-group comparisons of categorical variables. Patients were grouped in tertiles of CIMT. Receiver operator characteristics (ROC) curve analysis and the area under the curve (AUC) were used as a measure of the predictive accuracy of CIMT on the considered outcomes. The best cutoff of CIMT was selected by maximizing the sum of sensitivity and specificity. 
Survival analysis with Kaplan-Meier curves and log-rank test was performed to analyze tertiles in each outcome considered. Cox-proportional hazards models were built to assess the independent predictive value of CIMT both in univariate and multivariate models. Two risk prediction models were considered: (a) adjusted for age and gender (known predictors of CIMT); (b) adjusted for age, gender, and other significant predictors of outcome identified in univariate analysis. We also performed subgroup analysis to identify which specific subgroups might benefit of CIMT assessment.

\section{3 | RESULTS}

We included in the initial study 300 consecutive patients, who had been referred to our hospital from 2008 to 2010 for coronary
TABLE 1 Baseline characteristics and outcome by CIMT tertiles

\begin{tabular}{|c|c|c|c|c|c|}
\hline & $\begin{array}{l}\text { Tertile } 1 \\
\mathrm{IMT}<0.7 \\
\mathrm{n}=111\end{array}$ & $\begin{array}{l}\text { Tertile } 2 \\
\text { IMT } 0.7-0.9 \\
\mathrm{n}=98\end{array}$ & $\begin{array}{l}\text { Tertile } 3 \\
\text { IMT } \geq 0.9 \\
n=87\end{array}$ & $\begin{array}{l}\text { Total } \\
n=296\end{array}$ & $P$-value \\
\hline Age (years) & $61 \pm 10$ & $66 \pm 8$ & $67 \pm 8$ & $65 \pm 9$ & $<0.001$ \\
\hline Male gender (\%) & 37.8 & 57.1 & 73.6 & 54.7 & $<0.001$ \\
\hline $\mathrm{BMI}\left(\mathrm{kg} / \mathrm{m}^{2}\right)$ & $28 \pm 4$ & $29 \pm 5$ & $28 \pm 3$ & $28 \pm 4$ & 0.413 \\
\hline MS (\%) & 55.0 & 52.0 & 60.9 & 55.7 & 0.468 \\
\hline HTN (\%) & 73.9 & 80.6 & 85.1 & 79.4 & 0.145 \\
\hline Smoking (\%) & 5.4 & 9.2 & 13.8 & 9.1 & 0.126 \\
\hline Hyperlipidemia (\%) & 77.5 & 65.3 & 65.5 & 69.9 & 0.090 \\
\hline Diabetes (\%) & 15.3 & 25.5 & 25.3 & 21.6 & 0.124 \\
\hline Statins (\%) & 56.8 & 60.2 & 64.4 & 60.1 & 0.555 \\
\hline CAD (\%) & 27.0 & 44.9 & 56.3 & 41.6 & $<0.001$ \\
\hline $\begin{array}{l}\text { Total cholesterol } \\
(\mathrm{mg} / \mathrm{dL})\end{array}$ & $186 \pm 48$ & $189 \pm 46$ & $181 \pm 40$ & $185 \pm 44$ & 0.405 \\
\hline $\begin{array}{l}\text { LDL-cholesterol } \\
\text { (mg/dL) }\end{array}$ & $117 \pm 37$ & $119 \pm 38$ & $116 \pm 30$ & $117 \pm 35$ & 0.831 \\
\hline $\begin{array}{l}\text { HDL-cholesterol } \\
(\mathrm{mg} / \mathrm{dL})\end{array}$ & $46 \pm 14$ & $48 \pm 12$ & $41 \pm 11$ & $45 \pm 13$ & 0.002 \\
\hline $\begin{array}{l}\text { Triglycerides (mg/ } \\
\mathrm{dL} \text { ) }\end{array}$ & $114 \pm 86$ & $114 \pm 71$ & $114 \pm 62$ & $114 \pm 74$ & 0.999 \\
\hline HbA1c (\%) & $6.5 \pm 0.9$ & $6.9 \pm 1.3$ & $6.9 \pm 1.5$ & $6.7 \pm 1.2$ & 0.050 \\
\hline Creatinine (mg/dL) & $0.9 \pm 0.3$ & $0.9 \pm 0.2$ & $1.0 \pm 0.3$ & $0.9 \pm 0.3$ & 0.242 \\
\hline $\mathrm{PCl}$ at index (\%) & 19.8 & 31.6 & 31.0 & 27.0 & 0.096 \\
\hline CABG at index (\%) & 0.9 & 5.1 & 9.2 & 4.7 & 0.024 \\
\hline $\begin{array}{l}\text { Revascularization at } \\
\text { index (\%) }\end{array}$ & 20.7 & 36.7 & 40.2 & 31.8 & 0.006 \\
\hline $\begin{array}{l}\text { All-cause mortality } \\
\text { (\%) }\end{array}$ & 5.4 & 13.3 & 12.6 & 10.1 & 0.112 \\
\hline $\begin{array}{l}\text { Composite outcome } \\
\text { (\%) }\end{array}$ & 14.4 & 25.5 & 27.6 & 22.0 & 0.049 \\
\hline $\begin{array}{c}\text { Cardiovascular } \\
\text { mortality (\%) }\end{array}$ & 0.9 & 4.1 & 6.9 & 3.7 & 0.084 \\
\hline ACS (\%) & 3.6 & 4.1 & 4.6 & 4.1 & 0.940 \\
\hline $\mathrm{PCl}(\%)$ & 5.4 & 6.1 & 6.9 & 6.1 & 0.909 \\
\hline CABG (\%) & 0 & 1.0 & 1.1 & 0.7 & 0.543 \\
\hline Stroke/TIA (\%) & 1.8 & 1.0 & 4.6 & 2.4 & 0.247 \\
\hline Heart failure (\%) & 2.7 & 5.1 & 3.4 & 3.7 & 0.650 \\
\hline $\begin{array}{l}\text { Cardiovascular } \\
\text { admission (\%) }\end{array}$ & 11.7 & 14.3 & 17.2 & 14.2 & 0.542 \\
\hline
\end{tabular}

$\mathrm{ACS}$ = acute coronary syndrome; $\mathrm{BMI}=$ body mass index; $\mathrm{CABG}$ = coronary artery bypass grafting; $\mathrm{CAD}=$ coronary artery disease; $\mathrm{CIMT}=$ carotid intima-media thickness; $\mathrm{HTN}=$ hypertension; $\mathrm{MS}=$ metabolic syndrome; $\mathrm{PCI}=$ percutaneous coronary intervention; $\mathrm{TIA}=$ transient ischemic attack. 
angiography, aged 38-86 years. Four patients were lost during follow-up, and our final study sample included 296 patients. Mean age at entry was $65 \pm 9$ years, $55 \%$ males. Median follow-up was $6.9 \pm 2.2$ years.

With increasing CIMT tertile, the prevalence of significant coronary artery disease also increased, as well as the rate of revascularization at the index hospitalization (Table 1). The increase in CIMT was associated with age and male gender, but not with other cardiovascular risk factors. We found, however, some trend for relationship with hypertension, smoking, and diabetes and a paradoxical relationship with hyperlipidemia. HDL-cholesterol was lower in the group with the highest CIMT and there was a trend for higher $\mathrm{HbA1c}$ in both tertiles with CIMT $>0.7 \mathrm{~mm}$.

Patients in the tertile of higher CIMT had a trend for more frequent all-cause mortality (log-rank, $P=0.105$ ), and for the composite outcome (Log-rank, $P=0.079$ ), particularly cardiovascular mortality and stroke/TIA (Table 1, Figure 1). CIMT had a modest predictive accuracy for both all-cause mortality (AUC 0.643, 95\% Cl 0.5430.743 ) and for the composite outcome (AUC 0.611, 95\% Cl 0.5340.688). The best cutoff of CIMT was $0.85 \mathrm{~mm}$, with a sensitivity of $70 \%$ and a specificity of $53 \%$ for all-cause mortality and a sensitivity of $66 \%$ and specificity of $55 \%$ for the composite outcome.

In univariate analysis, CIMT was a predictor of all-cause mortality as a continuous variable (Figure 2), with a 1.12 times the risk for each $0.1 \mathrm{~mm}$ increase in thickness, and also with the cutoff of $0.85 \mathrm{~mm}$, but not after adjustment (Table 2). For the composite outcome, in univariate analysis, it was also a predictor (both as categorical and continuous distribution) (Figure 2) but no longer after adjustment in multivariate models (Table 3). The other independent predictors of outcome were diabetes and the presence of coronary artery disease that were included in model 2 for further adjustment.
In the subgroup analysis, CIMT assessment (with the cutoff of $0.85 \mathrm{~mm}$ ) was useful to assess the risk of all-cause mortality in patients with hypertension, hyperlipidemia, and metabolic syndrome and in nondiabetic patients (Figure 3). For the composite outcome, CIMT assessment was useful in females, patients with hypertension, hyperlipidemia, and metabolic syndrome, and also in nonsmokers, patients without diabetes, and patients without coronary artery disease (Figure 4).

\section{4 | DISCUSSION}

The results presented herein confirmed the previously described association between CIMT and age (related to "vascular age") as well as with gender. ${ }^{8,9} \mathrm{CIMT}$ is a predictor of outcome, but after adjustment for other variables, particularly age and gender, this association is lost. However, with the use of a specific cutoff of $0.85 \mathrm{~mm}$, the evaluation of CIMT might be useful in specific subsets of patients, both for all-cause mortality and for the composite outcome.

Previously published prospective studies on CIMT and cardiovascular disease risk demonstrated that CIMT was significantly associated with risk for myocardial infarction, stroke, coronary heart disease death, or a combination of these. ${ }^{7,8,11-19}$

The CAFES-CAVE study in healthy individuals showed that increased wall thickness was associated with more vascular events (stroke and cardiovascular). ${ }^{12}$ The ARIC study with long-term follow-up (4-7 years) of asymptomatic individuals showed an increased risk of coronary heart disease incidence and stroke, particularly in women. ${ }^{13,14}$ In the Cardiovascular Health Study (in patients older than 65 years but without a history of cardiovascular admission), CIMT was directly associated with an increased risk of myocardial
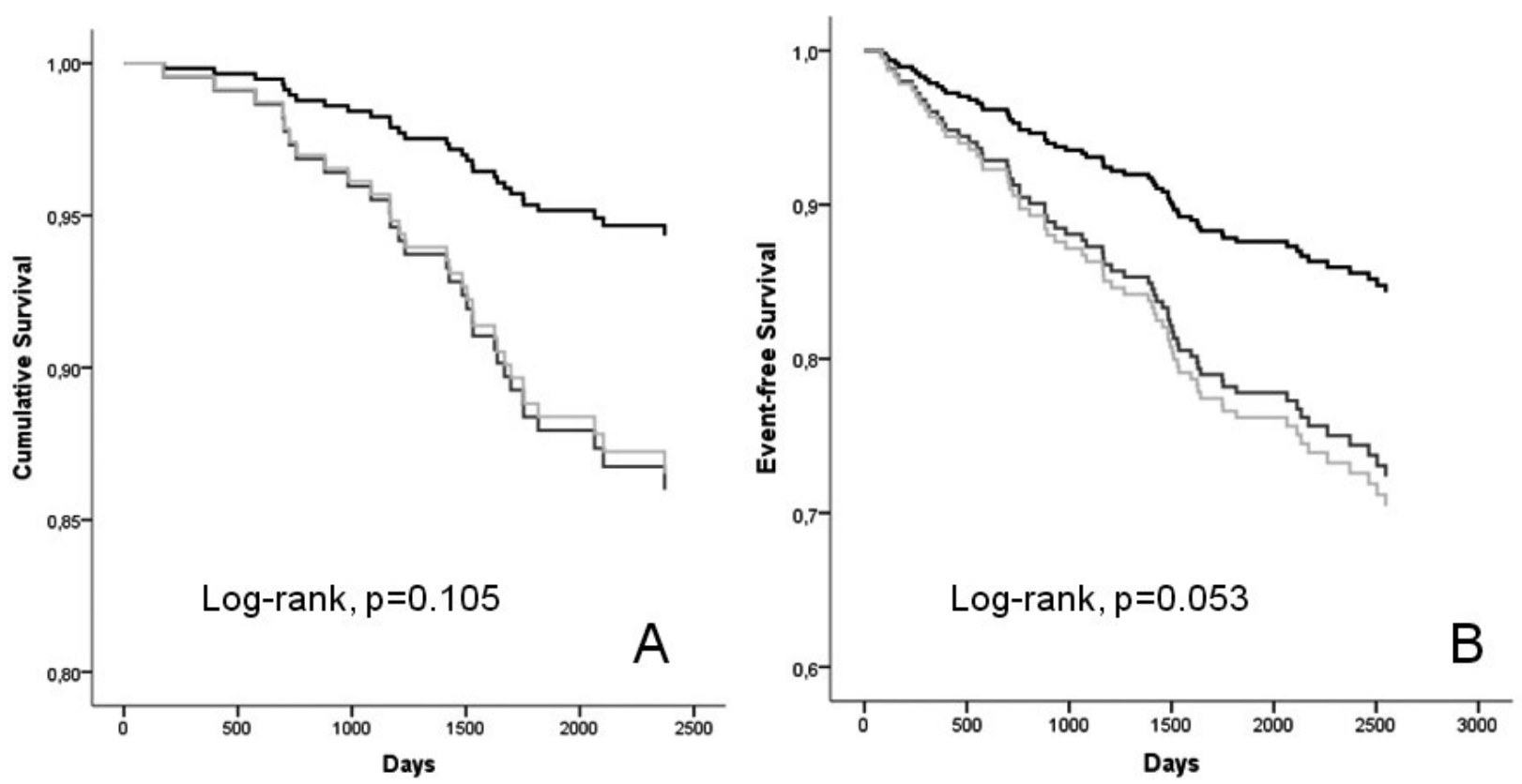

FIG URE 1 Kaplan-Meier curves for all-cause mortality (A) and composite outcome (B) according to carotid intima-media thickness tertiles 

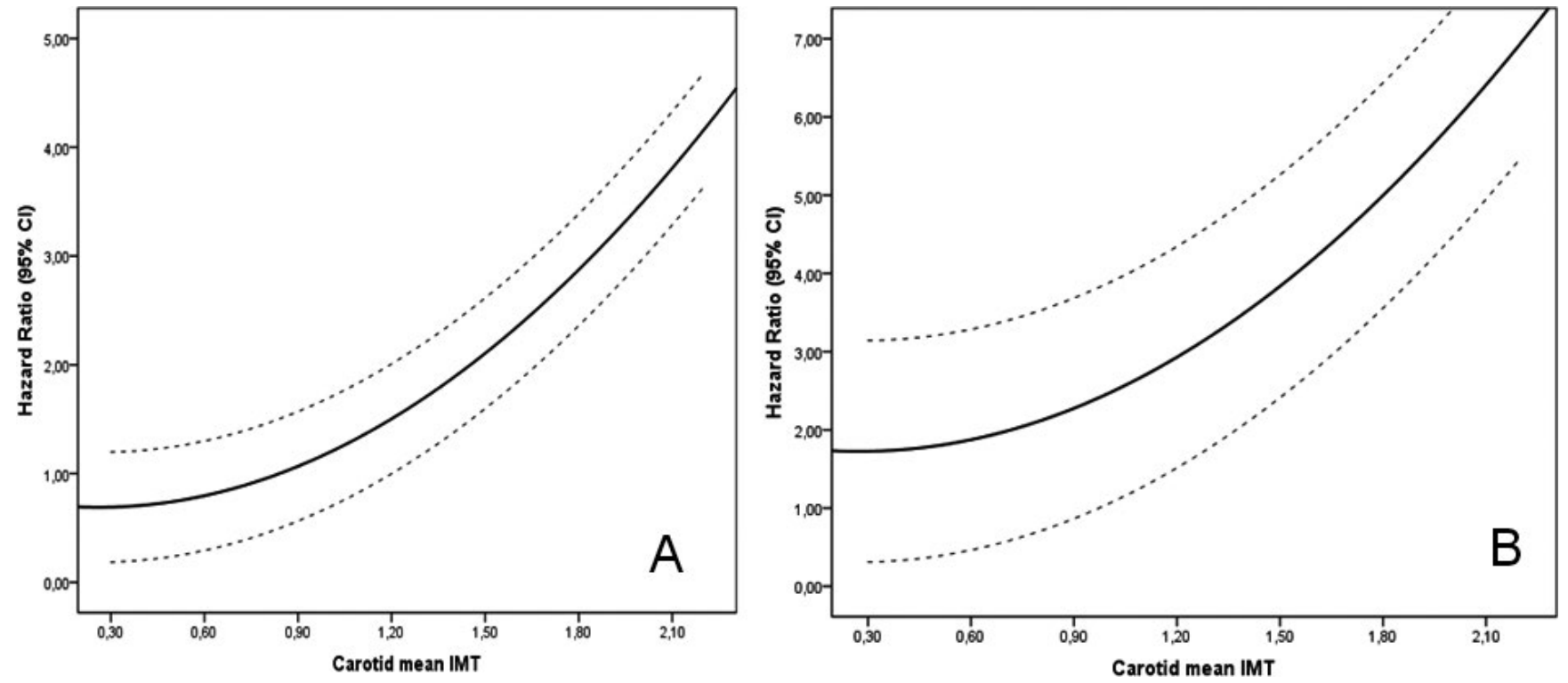

FIGURE 2 Hazard ratios (univariate) for all-cause mortality (A) and composite outcome (B) for each increase in carotid intima-media thickness

TAB LE 2 Univariate and multivariate analysis for all-cause mortality

\begin{tabular}{|llll|}
\hline & Hazard ratio & $95 \% \mathrm{Cl}$ & $P$-value \\
\hline Univariate & & & \\
\hline Tertile 1 & Reference & - & - \\
\hline Tertile 2 & 2.59 & $0.98-6.82$ & 0.054 \\
\hline Tertile 3 & 2.49 & $0.92-6.73$ & 0.072 \\
\hline Continuous IMT & 1.12 & $1.01-1.23$ & 0.029 \\
\hline CIMT $\geq 0.85 \mathrm{~mm}$ & 2.56 & $1.17-5.59$ & 0.018 \\
\hline Model 1 & & & \\
\hline Tertile 1 & Reference & - & - \\
\hline Tertile 2 & 1.77 & $0.67-4.67$ & 0.251 \\
\hline Tertile 3 & 1.38 & $0.50-3.81$ & 0.528 \\
\hline Continuous IMT & 1.07 & $0.95-1.20$ & 0.250 \\
\hline CIMT $\geq 0.85 \mathrm{~mm}$ & 1.49 & $0.66-3.35$ & 0.336 \\
\hline Model 2 & & & \\
\hline Tertile 1 & Reference & - & - \\
\hline Tertile 2 & 1.51 & $0.56-4.04$ & 0.416 \\
\hline Tertile 3 & 1.25 & $0.45-3.46$ & 0.668 \\
\hline Continuous IMT & 1.07 & $0.95-1.22$ & 0.248 \\
\hline CIMT $\geq 0.85 \mathrm{~mm}$ & 1.35 & $0.58-3.14$ & 0.481 \\
\hline
\end{tabular}

Model 1: Adjusted for age and gender; model 2: adjusted for age, gender, coronary artery disease, and diabetes. Continuous IMT for each $0.1 \mathrm{~mm}$ increase.

$\mathrm{Cl}=$ confidence interval; IMT = mean carotid intima-media thickness.

infarction and stroke. ${ }^{15}$ The Rotterdam Study showed similar results, but a more modest risk. ${ }^{16}$ Additional adjustment for age and gender attenuated the association, particularly for coronary heart disease
TAB LE 3 Univariate and multivariate analysis for the composite outcome

\begin{tabular}{|llll}
\hline & Hazard ratio & $95 \% \mathrm{Cl}$ & P-value \\
\hline Univariate & & & \\
\hline Tertile 1 & Reference & - & - \\
\hline Tertile 2 & 1.90 & $1.01-3.55$ & 0.046 \\
\hline Tertile 3 & 2.06 & $1.09-3.87$ & 0.026 \\
\hline Continuous IMT & 1.09 & $1.01-1.17$ & 0.022 \\
\hline CIMT $\geq 0.85 \mathrm{~mm}$ & 2.19 & $1.31-3.66$ & 0.003 \\
\hline Model 1 & & & \\
\hline Tertile 1 & Reference & - & - \\
\hline Tertile 2 & 1.43 & $0.75-2.70$ & 0.272 \\
\hline Tertile 3 & 1.36 & $0.71-2.62$ & 0.361 \\
\hline Continuous IMT & 1.42 & $0.84-2.39$ & 0.190 \\
\hline CIMT $\geq 0.85 \mathrm{~mm}$ & 1.52 & $0.88-2.62$ & 0.131 \\
\hline Model 2 & & & \\
\hline Tertile 1 & Reference & - & - \\
\hline Tertile 2 & 1.11 & $0.58-2.13$ & 0.750 \\
\hline Tertile 3 & 0.99 & $0.50-1.94$ & 0.972 \\
\hline Continuous IMT & 1.02 & $0.94-1.11$ & 0.622 \\
\hline CIMT $\geq 0.85 \mathrm{~mm}$ & 1.15 & $0.65-2.02$ & 0.629 \\
\hline & & & \\
\hline
\end{tabular}

Model 1: Adjusted for age and gender; model 2: adjusted for age, gender, and diabetes. Continuous IMT for each $0.1 \mathrm{~mm}$ increase.

$\mathrm{Cl}=$ confidence interval; IMT = mean carotid intima-media thickness

events and less for stroke. The major impact was only observed for higher levels of CIMT, especially $\geq 1 \mathrm{~mm}$. The Carotid Atherosclerosis Progression Study (CAPS) showed similar results. ${ }^{18}$ Finally, a metaanalysis showed a hazard ratio for myocardial infarction adjusted for 
$\mathrm{HR}(95 \% \mathrm{CI}) \quad$ p-value for interaction

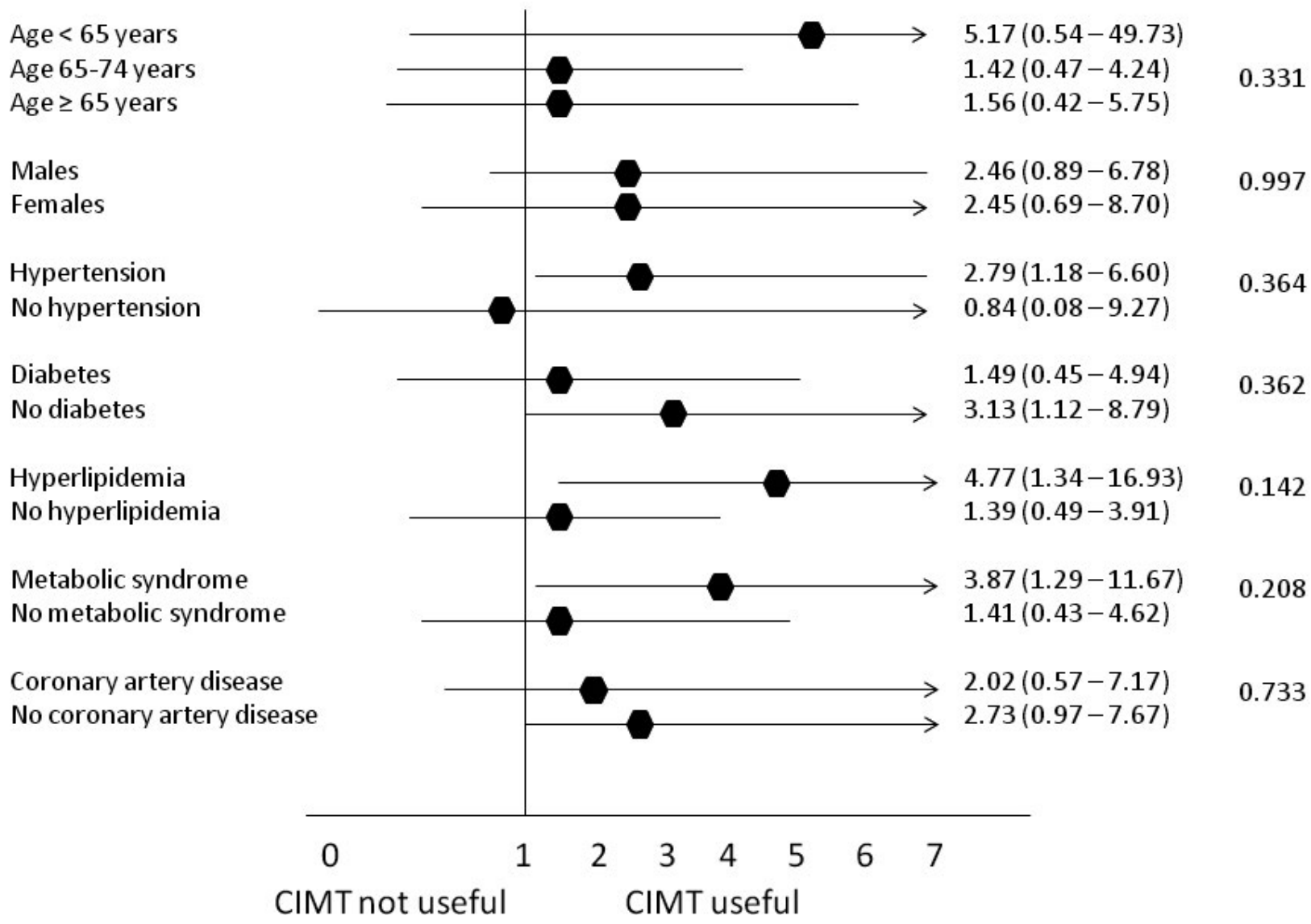

FIGURE 3 Subgroup analysis of carotid intima-media thickness $\geq 0.85 \mathrm{~mm}$ for all-cause mortality

age and gender of $1.26(1.21-1.30)$ and $1.32(1.27-1.38)$ for stroke for each $0.1 \mathrm{~mm}$ increase in CIMT. ${ }^{19}$

More recent studies showed different results. Both the Tromso study and the Multi-Ethnic Study of Atherosclerosis (MESA) showed that common CIMT is associated with incident stroke in unadjusted analysis, but this association became nonsignificant after adjustment for several risk factors. ${ }^{20,21}$ In the MESA study, measurements of CMIT were made in a more proximal part of the common carotid and more distant to the carotid bulb. ${ }^{20}$ In the Tromso study, measurements that included the carotid bifurcation were associated with stroke. ${ }^{21}$ These discrepancies confirm the importance of site measurement.

Carotid intima-media thickness value also provides additional information beyond traditional risk factors for classifying patients in regard to the likelihood of the presence of significant angiographic coronary artery disease. ${ }^{22}$ These findings provide support to the concept that CIMT measurements can be used as a surrogate marker of atherosclerosis. A report from the ARIC study demonstrated the ability of carotid ultrasound data to improve coronary heart disease risk prediction. ${ }^{22}$ Thus, ultrasound-based CIMT measurement and identification of plaque presence improve coronary heart disease risk prediction and should be considered in the intermediate-risk group. However, intima-media thickening is a feature of arterial wall aging, and carotid wall thickening is not synonymous of atherosclerosis, particularly in the absence of plaques. It represents the pathophysiologic substrate that explains why CIMT is a risk factor and a marker of cardiovascular risk. Our previous work showed that age is one of the main independent predictors of $\mathrm{CIMT}^{8,9}$

Fewer studies addressed the prognostic impact of CIMT in patients with established coronary artery disease or high cardiovascular risk and thus recommendations for this population about the use of CIMT study is not well established. The Angina Prognosis Study in Stockholm (APSIS) study in patients with stable angina showed that CIMT is a weak predictor of events, with carotid plaques being highly predictive. ${ }^{6}$ However, median follow-up was only of 3 years. Another study in patients with nonST-acute coronary syndromes showed that CIMT was correlated with TIMI risk score but it did not predict 6-month clinical outcome. $^{7}$ This correlation with TIMI risk score was mainly driven by the association demonstrated of CIMT with age and diabetes, main components of TIMI risk score. These results are in line with our own results.

Surprisingly, we found no association between CIMT and both total cholesterol and LDL-cholesterol. The same was previously reported in the ISMIR study. ${ }^{23}$ In that study, in the metabolic syndrome population, only triglycerides remained significantly associated with CIMT value, while total cholesterol and non-HDL-cholesterol failed 
Age $<65$ years Age 65-74 years

Age $\geq 65$ years

Males

Females

Hypertension

No hypertension

Diabetes

No diabetes

Hyperlipidemia

No hyperlipidemia

Smoking

No smoking

Metabolic syndrome

No metabolic syndrome

Coronary artery disease

No coronary artery disease

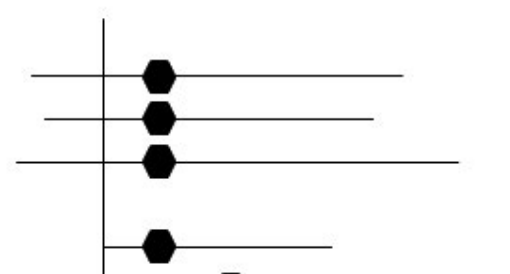

$1.76(0.70-4.43)$

$1.85(0.84-4.05)$

$1.74(0.57-5.33)$

$1.87(0.98-3.59)$

$2.50(1.07-5.86)$

0.595

$2.13(1.22-3.72)$

$1.78(0.45-7.12)$

0.054

$1.10(0.48-2.55)$

$2.80(1.46-5.40)$

0.090

$2.73(1.38-5.41)$

$1.48(0.67-3.26)$

0.269

$1.30(0.14-12.58)$

$2.32(1.37-3.95)$

0.652

$2.48(1.26-4.88)$

$1.80(0.81-4.01)$

0.521

$1.09(0.56-2.15)$

$2.75(1.23-6.12)$

0.088

\section{$\begin{array}{llllllll}0 & 1 & 2 & 3 & 4 & 5 & 6 & 7\end{array}$ \\ CIMT not useful CIMT useful}

FIGURE 4 Subgroup analysis of carotid intima-media thickness $\geq 0.85 \mathrm{~mm}$ for the composite outcome

to reach a significant correlation. The author's explanation was that statin treatment, present in almost half of the subgroups, could alter the typical lipid profile of these patients, particularly regarding cholesterol levels, and can thus modify the relationship between plasma cholesterol and CIMT. Our patients also had a very high rate of statin therapy, and lipid profile was better than expected, which might have influenced our results. In fact, only HDL-cholesterol showed some inverse relationship with CIMT.

Carotid intima-media thickness has a relatively low sensitivity and specificity for the prediction of cardiovascular outcomes, and after adjustment for other independent predictors, it is no longer an independent predictor. However, we observed that in specific subsets of patients it might be useful for the prediction of all-cause mortality, particularly in patients with hypertension, hyperlipidemia, and metabolic syndrome and in nondiabetic patients, and also in females, nonsmokers, and patients without coronary artery disease for the composite outcome. This is easily explained by the fact that older age and male gender are closely associated with increases in CIMT and cardiovascular events, and thus, it does not add significant prognostic information. The same is true for diabetes, smoking, and coronary artery disease, all of which are known predictors of cardiovascular events and the benefit of CIMT assessment is less important for risk assessment.

\section{1 | Limitations}

The scanning protocol for CIMT measurement only focused on the common carotid artery due to difficulties in adequate evaluation of CIMT within the bulb and internal carotid arteries. Scanning of the remaining segments of the carotid arteries might be important to avoid missing "upstream" advanced atherosclerosis. The site of measurement has important implications as already mentioned but we used the recommended technique.

Manual assessment of CIMT is not optimally reproducible. There have been rapid advances in ultrasound technology resulting in greater consistency and resolution of images. Semi-automated edgedetection software to measure CIMT has been developed and has been found to be both accurate and reproducible. At the time of data collection, we did not have the automated software, but our reproducibility analysis is quite good, better than what was reported by Van der Meer. ${ }^{17}$ Automated technology might have yield different results.

Angiographic study does not assess directly coronary plaque morphology. Intravascular ultrasound allows cross-sectional imaging of coronary arteries and provides more comprehensive assessment of atherosclerotic plaque in vivo and this is considered the gold standard technique. For that reason, quantitative coronary angiography analysis might be slightly misleading. 
The sample size is somewhat small but we considered that the original population was an important group and decided to perform long-term follow-up in this cohort of patients. A study in a larger sample is advisable to confirm our results. It would also have been interesting to include a low-risk group for comparison, but the original work was focused in patients with high cardiovascular risk and thus we do not have a population of patients with low cardiovascular risk, CIMT assessment, and such a long-term follow-up for comparison.

\section{5 | CONCLUSIONS}

Patients with higher CIMT have worst long-term outcome both for fatal and nonfatal cerebral and cardiovascular events. However, this is mainly driven by age and gender. Measurements of CIMT might be useful in specific subsets of patients, particularly in patients with hypertension, hyperlipidemia, and metabolic syndrome and in nondiabetic patients.

\section{ACKNOWLEDGMENTS}

The authors wish to thank Professor Maria Pedro Guarino for the English review of the manuscript.

\section{ORCID}

Ana Teresa Timóteo (iD http://orcid.org/0000-0001-6061-9663

\section{REFERENCES}

1. GBD 2016 Causes of Death Collaborators. Global, regional, and national age-sex specific mortality for 264 causes of death. 19802016: a systematic analysis for the Global Burden of Disease Study 2016. Lancet 2017;390:1151-1210.

2. World Health Organization (WHO). The top 10 causes of death. Fact sheet $\mathrm{n}^{\circ} 310$. Available in: http://www.who.int/mediacentre/ factsheets/fs310/en/. Assessed April 10, 2016.

3. Programa Nacional para as doenças cérebro-cardiovasculares 2017. Ed. Direção Geral Saúde, Lisboa, September 2017, 5.

4. Townsend N, Wilson L, Bhatnagar P, et al. Cardiovascular diseases in Europe: epidemiological update 2016.EurHeartJ.2016;37:3232-3245.

5. Society of Atherosclerosis Imaging and Prevention Developed in collaboration with the International Atherosclerosis Society. Appropriate use criteria for carotid intima-media thickness testing. Society of Atherosclerosis Imaging and Prevention. Atherosclerosis. 2011;214:43-46.

6. Held C, Hjemdahl P, Eriksson I, et al. Prognostic implications of intima-media thickness and plaques in the carotid and femoral arteries in patients with stable angina pectoris. Eur Heart $J$ 2001;22:62-72.

7. Tello-Montoliu A, Moltó JM, López-Hernandez N, et al. Common carotid artery intima-media thickness and intracranial pulsatility index in non-ST-elevation acute coronary syndromes. Cerebrovasc Dis. 2007;24:338-342.

8. Timóteo AT, Mota Carmo M, Ferreira RC. Can metabolic syndrome presence predict carotid intima-media thickness? J Clin Hypertens (Greenwich). 2012;14:507-513.
9. Timóteo AT, Mota Carmo M, Ferreira RC. Carotid intima-media thickness and carotid plaques improves prediction of obstructive angiographic coronary artery disease in women. Angiology. 2013;64:57-63.

10. Touboul PJ, Hennerici MG, Meairs S, et al. Mannheim carotid intimamedia thickness Consensus. Cerebrovac Dis. 2004;18:346-349.

11. Kotsis VT, Pitiriga VC, Stabouli SV, et al. Carotid artery intima-media thickness could predict the presence of coronary artery lesions. Am J Hypertens. 2005;18:601-606.

12. Belcaro G, Nicolaides AN, Ramaswami G, et al. Carotid and femoral ultrasound morphology screening and cardiovascular events in low risk subjects: a 10-year follow-up study (the CAFES-CAVE Study). Atherosclerosis. 2001;156:379-387.

13. Chambless LE, Heiss G, Folsom AR. Association of coronary heart disease incidence with carotid arterial wall thickness and major risk factors: the Atherosclerosis Risk in Communities (ARIC) Study. 1987-1993. Am J Epidemiol. 1997;146:483-494.

14. Chambless LE, Folsom AR, Clegg LX. Carotid wall thickness is predictive of incident clinical stroke; The Atherosclerotic Risk in Communities (ARIC) Study. Am J Epidemiol. 2000;151:478-487.

15. O'Leary DH, Polak JF, Kronmal RA, et al. Carotid artery intima and media thickness as a risk factor for myocardial infarction and stroke in older adults. N Engl J Med. 1999;340:14-22.

16. Bots ML, Hoes AW, Koudstall PJ, et al. Common carotid intimamedia thickness and risk of stroke and myocardial infarction: the Rotterdam study. Circulation. 1997;96:1432-1437.

17. Van der Meer IM, Bots ML, Hofman A, et al. Predictive value of non-invasive measures of atherosclerosis for incident myocardial infarction. Circulation. 2004;109:1089-1094.

18. Lorenz MW, von Kegler S, Steinmetz H, et al. Carotid intima-media thickness indicates a higher vascular risk across a wide age range: prospective data from the Carotid Atherosclerosis Progression Study (CAPS). Stroke. 2006;37:87-92.

19. Lorenz MW, Markus HS, Bots ML, et al. Prediction of clinical cardiovascular events with carotid intima-media thickness. A systematic review and meta-analysis. Circulation. 2007;115:459-467.

20. Polak JF, Pencina MJ, O'Leary DH, D'Agostino RB. Common carotid artery intima-media thickness progression as a predictor of stroke in Multi-Ethnic Study of Atherosclerosis. Stroke. 2011;42:3017-3021.

21. Mathiesen EB, Johnsen SH, Wilsgaard T, et al. Carotid plaque area and intima-media thickness in prediction of first-ever ischemic stroke: a 10-year follow-up of 6584 men and women. The Tromson Study. Stroke. 2011;42:972-978.

22. Nambi V, Chambless L, Folsom AR, et al. Carotid intima-media thickness and presence or absence of plaque improves prediction of coronary heart disease risk in the Atherosclerosis Risk in Communities (ARIC) study. J Am Coll Cardiol. 2010;55:1600-1607.

23. Antonini-Canterin F, La Carrubba S, Gullace G, et al. Association between carotid atherosclerosis and metabolic syndrome: results from the ISMIR Study. Angiology. 2010;61:443-448.

How to cite this article: Timóteo AT, Mota Carmo M, Soares C, Ferreira RC. Has carotid intima-media thickness prognostic impact in patients with high cardiovascular risk? A long-term cohort study. Echocardiography. 2019;36:125-132. https://doi. org/10.1111/echo.14207 